\title{
Body-related discrimination and dieting and substance use behaviors in adolescence
}

\author{
Angelina R. Sutin ${ }^{\mathrm{a}, *}$, Yannick Stephan ${ }^{\mathrm{b}}$, Eric Robinson ${ }^{\mathrm{c}}$, Michael Daly ${ }^{\mathrm{d}}$, Antonio Terracciano ${ }^{\mathrm{a}}$ \\ ${ }^{\text {a }}$ Florida State University College of Medicine, USA \\ ${ }^{\mathrm{b}}$ Euromov University of Montpellier, France \\ ${ }^{c}$ University of Liverpool, UK \\ ${ }^{\mathrm{d}}$ Maynooth University, Ireland
}

A R T I C L E I N F O

\section{Keywords:}

Weight stigma

Body discrimination

Peer victimization

Dieting behavior

Substance use

\begin{abstract}
A B S T R A C T
Unfair treatment on the basis of a physical characteristic, such as body weight, is associated with unhealthy dieting behaviors in adolescence and adulthood and has also been implicated in substance use. Peer victimization is likewise associated with these health-risk behaviors. It is unclear, however, whether body discrimination is associated with these behaviors independent of peer victimization. The present research uses data from the Longitudinal Study of Australian Children (LSAC) to test the relation between body discrimination and dieting and substance use behaviors in adolescence and test whether the associations are independent of peer victimization, as well as depressive symptoms which are associated with both forms of victimization and healthrisk behaviors. Participants $(N=2955)$ reported on body discrimination, dieting behaviors, and substance use at ages 14-15. Participants who experienced body discrimination were more likely to report fear of gaining weight, losing control over eating, going without eating, using medicine or vomiting to control their weight, engaging in restrained eating, and exercising to control their weight. They also had tried nicotine, alcohol, and marijuana. The associations with eating and alcohol use were independent of peer victimization, whereas the associations with smoking and marijuana were reduced when peer victimization was included in the model. All associations were also independent of depressive symptoms. Overall, the findings suggest that body discrimination is associated with harmful health behaviors at least as early as age 14 .
\end{abstract}

Unfair treatment because of a body's physical characteristics is a relatively common experience (Puhl, Andreyeva, \& Brownell, 2008). Body discrimination includes unfair treatment on the basis of various aspects of the body, such as weight, height, and body shape. Such experiences have been associated with health-risk behaviors and poor physical and mental health outcomes. In adolescence, for example, teasing because of appearance is associated with lower feelings of selfworth and more symptoms of anxiety and depression (Duncan, ZimmerGembeck, \& Furman, 2019) and more appearance-specific anxiety symptoms (Zimmer-Gembeck, Webb, Farrell, \& Waters, 2018); body teasing is also associated with a greater likelihood of being on a diet (Chisuwa-Hayami \& Haruki, 2017). Clinical samples of participants with bulimia nervosa or binge-eating disorder are more likely to have been teased because of their appearance compared to healthy controls (Lie, Rø, \& Bang, 2019). Further, adolescents who experience appearance discrimination tend to engage in more substance use (Klinck, Vannucci, Fagle, \& Ohannessian, 2020). The negative correlates of body-related discrimination do not end in adolescence. In adulthood, individuals who have experienced discrimination based on their appearance report worse self-rated health (Lee, Son, Yoon, \& Kim, 2017) and lower well-being (Sutin, Stephan, Carretta, \& Terracciano, 2015).

Body discrimination can occur for any number of physical characteristics, including weight, height, chest size, facial features, and/or body hair. Of these physical characteristics, most research has focused on the correlates of discrimination based on weight. Weight discrimination in adulthood is associated with behaviors conducive to obesity, including disordered eating and dieting-related behaviors (Tomiyama, 2014). Individuals who are treated unfairly because of their weight, for example, report that they binge eat, skip meals often, and engage in unhealthy methods for weight loss (e.g., use of laxatives) (Vartanian \& Porter, 2016). It has also been associated with psychological aspects of physical activity, including greater motivation to avoid physical activity (Vartanian \& Shaprow, 2008), and, when internalized, lower self-efficacy and less motivation to exercise (Pearl, Puhl, \&

\footnotetext{
* Corresponding author. Florida State University College of Medicine, 1115 W. Call Street, Tallahassee, FL, 32306, USA.

E-mail address: angelina.sutin@med.fsu.edu (A.R. Sutin).
} 
Dovidio, 2015). In addition to these behaviors conducive to obesity, weight discrimination has been associated with other high-risk healthrelated behaviors. Among adults, for example, those who experience weight discrimination report that they are current smokers, have recently driven under the influence of alcohol, and engage in high-risk sexual and/or substance use behavior (Sutin \& Terracciano, 2017). Of note, similar associations between weight discrimination and health outcomes are observed across measures that refer only to weight and measures that include other body characteristics (e.g., height) in addition to weight (Sutin, Stephan, \& Terracciano, 2015).

Similar to adults, adolescents are vulnerable to numerous types of victimization, including unfair treatment on the basis of their body (Pont, Puhl, Cook, \& Slusser, 2017). Also similar to adults, these experiences have been associated with behaviors conducive to obesity and other high-risk behaviors. Adolescents who have been teased because of their weight, for example, are more likely to engage in binge-eating behavior (Neumark-Sztainer et al., 2002). A meta-analysis likewise found weight teasing to be associated with more binge-eating behavior and greater dietary restraint (Menzel et al., 2010). Similar to adulthood, weight-based teasing is associated with lower self-efficacy and motivation for physical activity and somewhat less engagement in such activities (Greenleaf, Petrie, \& Martin, 2014). Other forms of peer victimization, such as bullying, have also been associated with numerous health-risk behaviors, including high-risk dieting behaviors and substance use: Adolescents who experience peer victimization are at greater risk of a range of disordered eating-related symptomology (Copeland et al., 2015) and risky substance use behaviors (Priesman, Newman, \& Ford, 2017). Further, the correlates of such victimization are long lasting. Adolescents who were bullied are at greater risk of obesity (Takizawa, Danese, Maughan, \& Arseneault, 2015) and substance use and other mental health disorders (Takizawa, Maughan, \& Arseneault, 2014) over twenty years later.

Individuals with overweight or obesity are particularly vulnerable to bullying and other forms of peer aggression (Puhl \& King, 2013). Previous research on the relation between peer victimization and health-risk behaviors in adolescence has generally focused on forms of body-based and more general aggression separately. Different forms of peer victimization tend to have similar correlates yet are distinct experiences. It is possible that any type of victimization is associated with engagement in worse health behaviors. From this perspective, the why and how of the victimization may not matter. It is also possible, however, that different types of victimization may have independent associations with risky behavior. In addition, both peer victimization in general and weight discrimination in particular have been associated with the experience of depressive symptoms (Moore et al., 2017; Spahlholz, Pabst, Riedel-Heller, \& Luck-Sikorski, 2016), which have also been implicated in health-risk behaviors (Earnshaw et al., 2017). As such, the association between weight discrimination and health-risk behaviors may also be due to depressive symptoms.

The purpose of the present research is to examine the dieting and substance use correlates of body discrimination in early adolescence and to test whether these associations are accounted for by peer victimization. We also control for depressive symptoms, given that low mood may be a confounder of the association between both body discrimination and peer victimization and the dieting and substance use outcomes (e.g., people with depressed affect may be more likely to report victimization and engage in more at-risk behaviors). We expect that adolescents who experience discrimination based on their body will be more likely to report engaging in dieting and disordered eatingrelated behaviors. We further expect that it will also be associated with substance use (cigarette smoking, alcohol consumption, use of marijuana). We test whether these associations are independent of peer victimization and whether the associations vary by gender and body mass index (BMI) category.

\section{Method}

\subsection{Participants and procedure}

Participants were drawn from the older (K) cohort of the Longitudinal Study of Australian Children (LSAC; http://data. growingupinaustralia.gov.au/index.html). LSAC was initiated in 2004 with two cohorts: a younger cohort (families were recruited into the study when the study child was an infant) and an older cohort (families were recruited into the study when the study child was 4 or 5). Families in both cohorts are re-interviewed every two years. As part of the inhome assessment at wave 6 of the older cohort, study children used an automated computer system to answer questions about many aspects of their lives. This approach allowed the adolescents to answer questions privately on a computer without fear that their answers would be overheard. The Australian Institute of Family Studies Ethics Committee approved data collection for LSAC and written informed consent for each studied family was obtained before family members were asked any questions. The Institutional Review Board at the Florida State University also approved analysis of these data.

Participants from the sixth wave of the older cohort were selected for the current analysis because this wave of this cohort was the first time the study children were asked about their experiences with discrimination. A total of up to 2955 participants (48\% female) had the requisite data to be included in the analysis. The sample sizes for the analyses ranged from 2889 to 2955 because of missing data across the outcomes.

\subsection{Measures}

\subsubsection{Predictors}

Body discrimination. Participants were asked, "In the last 6 months have you been treated unfairly or badly because of your body size, shape or physical appearance? (e.g., weight, height, chest size, body hair)." Participants responded yes (1) or no (0).

Peer victimization. Participants were asked a series of items that reflected various aspects of victimization (Vivolo-Kantor, Martell, Holland, \& Westby, 2014) that were derived from a scale to measure bullying at school (Brockenbrough, Cornell, \& Loper, 2002) and used in a previous longitudinal study of youth (McAra \& McVie, 2010). Specifically, participants were first told, "For the next questions, please think about things that might have happened to you at school, or out of school. Include texts, Facebook etc. as well as face-to-face contact. Do not include things that happened with your close family members (such as brothers and sisters)." Participants were then asked, "During the last 12 months, since [month at time of interview] last year ..." (1) someone hit or kicked me on purpose, (2) someone grabbed or shoved me on purpose, (3) someone threatened to hurt me, (4) someone threatened to take my things, (5) someone said mean things to me or called me names, (6) someone tried to keep others from being my friend, (7) someone did not let me join in what they were doing, (8) someone used force to steal something from me, (9) someone hurt me or tried to hurt me with a weapon, (10) someone stole my things to be mean to me, and (11) someone forced me to do something I didn't want to do. Participants responded yes or no to each item. These items were embedded in a section that asked participants about kids they spend time with, and since the items were derived from scales used to measure bullying, the items reflect experiences with other kids who are presumably their peers. We thus refer to this measure as peer victimization. Peer victimization was the sum of these eleven items (alpha $=.81$ ) and converted to $z$-scores (i.e., mean $=0$ and $S D=1$ ).

Depressive symptoms. Depressive symptoms were measured with the Short Mood and Feelings Questionnaire, which was developed to use in epidemiological surveys and has been found to correlate strongly with more in depth assessments (Angold et al., 1995). Participants rated 13 items about their mood (e.g., miserable or unhappy) in the last two 
weeks on a scale with $1=$ true, $2=$ sometimes, and $3=$ not true. Items were recoded to a scale that ranged from 0 [not true] to 2 [true], summed to create an index of depressive symptoms (range 0-26; alpha $=.94)$, and converted to $z$-scores.

\subsubsection{Outcomes}

Dieting behaviors. Participants were asked questions from the Branched Eating Test (Selzer, Hamill, Bowes, \& Patton, 1996) about dieting behaviors that occurred specifically within the last four weeks: participants were asked whether they had fear of gaining weight, had gone all day without eating, had lost control over their eating, had used medication to control their weight, had used vomiting to control their weight, and had exercised to lose weight. Participants responded yes or no to each item. Participants were also asked about their use of restrained eating strategies. Four items (e.g., "leave food at meal times to avoid putting on weight") were rated on a scale from 1 (seldom/never) to 4 (almost always/always). The mean was taken across these four items (alpha $=.83$ ).

Lifetime substance use. Cigarette smoking was measured with the item, "Have you ever smoked even part of a cigarette?" Alcohol consumption was measured with the item, "Have you ever had even part of an alcoholic drink?" Marijuana use was measured with the item, "Have you ever tried marijuana (cannabis, hash, grass, dope, weed, mull, yarndi, ganga, pot, a bong, a joint)?" Responses for each item were coded as yes (1) or no (0).

\subsubsection{Covariates}

BMI. Trained staff measured the height and weight of the study children. BMI $\left(\mathrm{kg} / \mathrm{m}^{2}\right)$ was converted to percentiles based on CDC growth charts (Kuczmarski et al., 2000) and categorized into underweight (BMI $<5$ th percentile), normal weight (BMI $\geq 5$ th percentile to $<85$ th percentile), overweight (BMI $\geq 85$ to $<95$ th percentile), and obese (BMI $\geq 95$ th percentile) categories. We entered these BMI categories into the analysis as three dummy-coded variables that compared underweight, overweight, and obese to normal weight (each dummy-coded variable was scored as 1 for the category and 0 for not the category; normal weight was the reference group).

Sociodemographic factors. Sociodemographic covariates included reported child gender ( $1=$ female, $0=$ male), child age (in years), indigenous status $(1=$ yes, $0=$ no), and household income (log transformed).

\subsection{Analytic strategy}

For each outcome, we tested three models: Model 1 tested the association between body discrimination and the outcomes, controlling for the sociodemographic covariates and BMI; Model 2 was Model 1 plus depressive symptoms; Model 3 was Model 2 plus peer victimization. The dieting behaviors were tested separately, as were the substance use outcomes. We used logistic regression for the dichotomous outcomes and linear regression for the one continuous outcome (restrained eating). Finally, we tested gender and BMI category as moderators of these associations to examine whether the associations with health-risk behaviors were stronger for adolescent girls than adolescent boys and whether the associations differed by BMI category.

\section{Results}

Descriptive statistics for all study variables are shown in Table 1. The correlation between body discrimination and peer victimization was $0.44(p<.01)$, which indicated that the two forms of harassment were related but distinct. Body discrimination in adolescence was associated with each of the dieting behaviors (Table 2): Participants who reported body discrimination reported that in the last four weeks they had feared gaining weight, lost control over their eating, had gone all day without eating, had used medicine or vomiting as a means of
Table 1

Descriptive statistics for the total sample and by body discrimination.

\begin{tabular}{|c|c|c|c|}
\hline \multirow[t]{2}{*}{ Variable } & \multirow{2}{*}{$\begin{array}{l}\text { Total Sample } \\
(N=2955)\end{array}$} & \multicolumn{2}{|c|}{ Body Discrimination } \\
\hline & & No $(n=2332)$ & Yes $(n=623)$ \\
\hline Gender (female) & $48 \%$ & $46.8 \%$ & $53.8 \%$ \\
\hline Age (years) & $14.40(.49)$ & $14.41(.49)$ & $14.37(.48)$ \\
\hline Indigenous status (yes) & $2 \%$ & $2 \%$ & $3 \%$ \\
\hline Household income $\mathrm{a}^{\mathrm{a}}$ & $\begin{array}{l}2650.63 \\
(2456.11)\end{array}$ & $\begin{array}{l}2705.90 \\
(2636.50)\end{array}$ & $\begin{array}{l}2443.74 \\
(1595.36)\end{array}$ \\
\hline Depressive symptoms $\mathrm{s}^{\mathrm{b}}$ & $5.35(6.53)$ & $4.25(5.805)$ & $9.46(7.39)$ \\
\hline \multicolumn{4}{|l|}{ Body mass index } \\
\hline Underweight & $6 \%$ & $6 \%$ & $7 \%$ \\
\hline Normal weight & $68 \%$ & $70 \%$ & $56 \%$ \\
\hline Overweight & $19 \%$ & $18 \%$ & $26 \%$ \\
\hline Obesity & $7 \%$ & $6 \%$ & $11 \%$ \\
\hline Peer victimization ${ }^{c}$ & $1.99(2.40)$ & $1.45(1.97)$ & $4.05(2.74)$ \\
\hline Body discrimination (yes) & $21 \%$ & $0 \%$ & $100 \%$ \\
\hline \multicolumn{4}{|l|}{ Dieting behaviors } \\
\hline Fear weight gain (yes) & $34 \%$ & $29 \%$ & $54 \%$ \\
\hline $\begin{array}{l}\text { Lost control over eating } \\
\text { (yes) }\end{array}$ & $31 \%$ & $26 \%$ & $48 \%$ \\
\hline $\begin{array}{l}\text { Gone all day without } \\
\text { eating (yes) }\end{array}$ & $7 \%$ & $5 \%$ & $15 \%$ \\
\hline $\begin{array}{l}\text { Medication to control } \\
\text { weight (yes) }\end{array}$ & $2 \%$ & $1 \%$ & $4 \%$ \\
\hline $\begin{array}{l}\text { Vomiting to control } \\
\text { weight (yes) }\end{array}$ & $2 \%$ & $1 \%$ & $5 \%$ \\
\hline $\begin{array}{l}\text { Exercise to control } \\
\text { weight (yes) }\end{array}$ & $48 \%$ & $45 \%$ & $60 \%$ \\
\hline Restrained eating ${ }^{\mathrm{d}}$ & $1.35(.53)$ & $1.28(.44)$ & $1.60(.73)$ \\
\hline \multicolumn{4}{|l|}{ Substance use } \\
\hline Ever smoke (yes) & $10 \%$ & $8 \%$ & $19 \%$ \\
\hline Ever tried alcohol (yes) & $52 \%$ & $49 \%$ & $66 \%$ \\
\hline $\begin{array}{l}\text { Ever tried marijuana } \\
\text { (yes) }\end{array}$ & $7 \%$ & $6 \%$ & $11 \%$ \\
\hline
\end{tabular}

Note. $N=2955 . n s$ ranges from 2952 to 2955 for the dieting behaviors because of missing data, and $n s$ range from 2886 to 2946 for substance use because of missing data. Values are means (standard deviations) or percentages. ${ }^{a}$ Household income per week is reported raw here for descriptive purposed. It was log transformed for the analyses. ${ }^{\mathrm{b}}$ Sum of depressive symptoms (possible range $0-26$ ). ${ }^{\mathrm{c}}$ Sum of peer victimization (possible range $0-11$ ). ${ }^{\mathrm{d}}$ Mean of four items rated on a scale from 1 (seldom/never) to 4 (almost always/always).

controlling their weight, and used physical activity to regulate their weight. These participants also reported more restrained eating (Table 3). Adolescents who reported body discrimination were at an up to two-fold increased risk of substance use (Table 4). These associations were apparent both controlling (Model 2) and not controlling (Model 1) for depressive symptoms.

We next examined the associations after further adjusting for peer victimization (Model 3, Tables 2-4). The associations between body discrimination and the dieting behaviors were reduced somewhat but remained significant. In contrast, the associations between body discrimination and cigarette smoking and marijuana use were reduced to non-significance; the association with alcohol consumption was reduced but remained significant.

Of note, all of the associations were independent of BMI category (Tables 2-4). Compared to normal weight, individuals in the overweight and obese weight categories generally shared similar associations with the dieting-related behaviors as body discrimination: Participants with overweight or obesity feared weight gain, lost control over their eating, and had gone all day without eating. They were not, however, more likely to engage in extreme forms of dieting-related behaviors, such as using medication or vomiting as a means to control weight. Further, the weight categories were either protective or unrelated to risk of substance use. This pattern suggests that the social experience of the body was independent and more harmful than measured adiposity. These associations were also independent of socio-demographic factors and depressive symptoms. 
Table 2

Logistic regression predicting dieting behaviors from body discrimination (Model 1) and controlling for depressive symptoms (Model 2) and peer victimization (Model 3).

\begin{tabular}{|c|c|c|c|c|c|c|c|c|c|}
\hline \multirow[t]{2}{*}{ Predictors } & \multicolumn{3}{|l|}{ Model 1} & \multicolumn{3}{|c|}{ Model 2} & \multicolumn{3}{|c|}{ Model 3} \\
\hline & OR & $95 \% \mathrm{CI}$ & $p$-value & OR & $95 \% \mathrm{CI}$ & $p$-value & OR & $95 \% \mathrm{CI}$ & $p$-value \\
\hline \multicolumn{10}{|l|}{$\underline{F e a r}$ of Weight Gain $^{a}$} \\
\hline Gender & 5.815 & $4.856-6.965$ & .000 & 5.414 & $4.506-6.505$ & .000 & 5.780 & $4.787-7.978$ & .000 \\
\hline Age & 1.119 & $.939-1.334$ & .209 & 1.106 & $.924-1.323$ & .271 & 1.113 & $.930-1.332$ & .242 \\
\hline Indigenous status & .781 & $.425-1.439$ & .428 & .640 & $.468-1.596$ & .640 & .846 & $.457-1.566$ & .595 \\
\hline Household income & 1.060 & $.939-1.197$ & .345 & 1.108 & $.978-1.255$ & .109 & 1.121 & $.988-1.271$ & .078 \\
\hline \multicolumn{10}{|l|}{ Body mass index } \\
\hline Underweight & .196 & $.117-.327$ & .000 & .201 & $.119-.339$ & .000 & .207 & $.122-.349$ & .000 \\
\hline Overweight & 2.058 & $1.661-2.550$ & .000 & 2.148 & $1.725-2.674$ & .000 & 2.152 & $1.727-2.682$ & .000 \\
\hline Obesity & 2.819 & $2.034-3.906$ & .000 & 2.804 & $2.005-3.922$ & .000 & 2.890 & $2.063-4.047$ & .000 \\
\hline Depressive symptoms & - & - & - & 1.583 & $1.446-1.733$ & .000 & 1.508 & $1.372-1.657$ & .000 \\
\hline Peer victimization & - & - & - & - & - & - & 1.222 & $1.104-1.353$ & .000 \\
\hline Body discrimination & 2.876 & $2.343-3.531$ & .000 & 2.081 & $1.672-2.590$ & .000 & 1.744 & $1.378-2.209$ & .000 \\
\hline Cox \& Snell $\mathrm{R}^{2}$ & $.177 / .205^{1}$ & & & .231 & & & .235 & & \\
\hline \multicolumn{10}{|c|}{ Lost Control Over Eating ${ }^{b}$} \\
\hline Gender & 3.175 & $2.678-3.765$ & .000 & 2.938 & $2.476-3.480$ & .000 & 3.206 & $2.684-3.831$ & .000 \\
\hline Age & .892 & $.751-1.058$ & .190 & .878 & $.738-1.045$ & .142 & .884 & $.742-1.052$ & .165 \\
\hline Indigenous status & .686 & $.375-1.252$ & .219 & .742 & $.404-1.360$ & .334 & .717 & $.389-1.323$ & .287 \\
\hline Household income & 1.054 & $.939-1.184$ & .371 & 1.089 & $.968-1.225$ & .157 & 1.107 & $.982-1.249$ & .096 \\
\hline \multicolumn{10}{|l|}{ Body mass index } \\
\hline Underweight & .240 & $.146-.393$ & .000 & .250 & $.152-.411$ & .000 & .258 & $.157-.425$ & .000 \\
\hline Overweight & 1.475 & $1.200-1.815$ & .000 & 1.501 & $1.217-1.851$ & .000 & 1.501 & $1.216-1.854$ & .000 \\
\hline Obesity & 1.738 & $1.269-2.380$ & .000 & 1.681 & $1.219-2.316$ & .002 & 1.748 & $1.266-2.415$ & .001 \\
\hline Depressive symptoms & - & - & - & 1.398 & $1.284-1.523$ & .000 & 1.306 & $1.195-1.428$ & .000 \\
\hline Peer victimization & - & - & - & - & - & - & 1.317 & $1.197-1.450$ & .000 \\
\hline Body discrimination & 2.54 & $2.098-3.095$ & .000 & 1.984 & $1.614-2.439$ & .000 & 1.558 & $1.245-1.948$ & .000 \\
\hline Cox \& Snell $\mathrm{R}^{2}$ & $.901 / .118^{1}$ & & & .135 & & & .144 & & \\
\hline Gone All Day Without & & & & & & & & & \\
\hline Gender & 1.714 & $1.280-2.294$ & .000 & 1.420 & $1.052-1.915$ & .022 & 1.517 & $1.118-2.057$ & .007 \\
\hline Age & .798 & $.592-1.075$ & .138 & .770 & $.569-1.043$ & .091 & .772 & $.570-1.047$ & .096 \\
\hline Indigenous status & .800 & $.309-2.072$ & .646 & .953 & $.361-2.510$ & .922 & .925 & $.349-2.453$ & .875 \\
\hline Household income & .853 & $.718-1.013$ & .070 & .900 & $.751-1.078$ & .252 & .912 & $.758-1.097$ & .328 \\
\hline Body mass index & & & & & & & & & \\
\hline Underweight & .553 & $.253-1.208$ & .137 & .617 & $.281-1.356$ & .229 & .646 & $.294-1.420$ & .277 \\
\hline Overweight & 1.404 & $.997-1.979$ & .052 & 1.433 & $1.010-2.033$ & .044 & 1.427 & $1.004-2.027$ & .047 \\
\hline Obesity & 2.275 & $1.462-3.542$ & .000 & 2.076 & $1.314-3.279$ & .002 & 2.132 & $1.346-3.378$ & .001 \\
\hline Depressive symptoms & - & - & - & 1.667 & $1.477-1.882$ & .000 & 1.590 & $1.399-1.807$ & .000 \\
\hline Peer victimization & - & - & - & - & - & - & 1.242 & $1.075-1.435$ & .003 \\
\hline Body discrimination & 2.835 & $2.114-3.802$ & .000 & 1.872 & $1.367-2.563$ & .000 & 1.505 & $1.061-2.133$ & .022 \\
\hline Cox \& Snell $\mathrm{R}^{2}$ & $.016 / .031^{1}$ & & & .051 & & & .054 & & \\
\hline Medication to Control & & & & & & & & & \\
\hline Gender & .750 & $.417-1.347$ & .335 & .663 & $.364-1.207$ & .179 & .834 & $.447-1.555$ & .568 \\
\hline Age & .583 & $.306-1.114$ & .102 & .562 & $.294-1.075$ & .082 & .566 & $.294-1.086$ & .087 \\
\hline Indigenous status & 1.639 & $.377-7.135$ & .510 & 1.827 & $.418-7.994$ & .424 & 1.750 & $.395-7.748$ & .461 \\
\hline Household income & .882 & $.622-1.250$ & .479 & .916 & $.639-1.313$ & .632 & .943 & $.642-1.386$ & .766 \\
\hline Body mass index & & & & & & & & & \\
\hline Underweight & .274 & $.037-2.042$ & .207 & .299 & $.040-2.229$ & .239 & .352 & $.047-2.628$ & .309 \\
\hline Overweight & .785 & $.367-1.680$ & .534 & .781 & $.365-1.674$ & .525 & .756 & $.350-1.631$ & .476 \\
\hline Obesity & 1.497 & $.633-3.542$ & .358 & 1.405 & $.590-3.343$ & .442 & 1.507 & $.625-3.635$ & .361 \\
\hline Depressive symptoms & - & - & - & 1.312 & $1.018-1.691$ & .036 & 1.104 & $.827-1.457$ & .502 \\
\hline Peer victimization & - & - & - & - & - & - & 1.717 & $1.322-2.230$ & .000 \\
\hline Body discrimination & 5.417 & $2.988-9.820$ & .000 & 4.407 & $2.353-8.254$ & .000 & 2.607 & $1.314-5.173$ & .006 \\
\hline Cox \& Snell $\mathrm{R}^{2}$ & $.003 / .014^{1}$ & & & .015 & & & .020 & & \\
\hline Vomiting to Control $\mathrm{W}$ & & & & & & & & & \\
\hline Gender & 7.902 & $3.095-20.177$ & .000 & 6.121 & $2.381-15.739$ & .000 & 6.699 & $2.581-17.329$ & .000 \\
\hline Age & .803 & $.430-1.499$ & .491 & .718 & $.379-1.359$ & .309 & .716 & $.377-1.362$ & .309 \\
\hline Indigenous status & 2.407 & $.676-8.575$ & .175 & 3.711 & $1.00-13.768$ & .050 & 3.517 & $.936-13.217$ & .063 \\
\hline Household income & .952 & $.614-1.475$ & .825 & 1.068 & $.684-1.667$ & .774 & 1.116 & $.711-1.750$ & .634 \\
\hline Body mass index & & & & & & & & & \\
\hline Underweight & .341 & $.046-2.543$ & .294 & .419 & $.055-3.162$ & .399 & .453 & $.060-3.431$ & .444 \\
\hline Overweight & .813 & $.389-1.700$ & .583 & .756 & $.355-1.610$ & .468 & .756 & $.354-1.616$ & .471 \\
\hline Obesity & .707 & $.208-2.403$ & .579 & .488 & $.136-1.746$ & .270 & .482 & $.133-1.743$ & .266 \\
\hline Depressive symptoms & - & - & - & 2.069 & $1.604-2.670$ & .000 & 1.925 & $1.466-2.257$ & .000 \\
\hline Peer victimization & - & - & - & - & - & - & 1.356 & $1.011-1.819$ & .042 \\
\hline Body discrimination & 6.671 & $3.568-12.473$ & .000 & 3.625 & $1.868-7.036$ & .000 & 2.692 & $1.296-5.591$ & .008 \\
\hline Cox \& Snell $\mathrm{R}^{2}$ & $.014 / .026$ & & & .036 & & & .037 & & \\
\hline Exercise to Control We & & & & & & & & & \\
\hline Gender & 1.954 & $1.678-2.276$ & .000 & 1.910 & $1.638-2.228$ & .000 & 2.004 & $1.174-2.344$ & .000 \\
\hline Age & 1.162 & $.996-1.357$ & .057 & 1.159 & $.992-1.353$ & .062 & 1.166 & $.998-1.362$ & .053 \\
\hline Indigenous status & .879 & $.512-1.509$ & .639 & .895 & $.521-1.537$ & .687 & .878 & $.510-1.512$ & .639 \\
\hline Household income & 1.067 & $.964-1.180$ & .209 & 1.074 & $.971-1.189$ & .166 & 1.083 & $.978-1.200$ & .126 \\
\hline
\end{tabular}


Table 2 (continued)

\begin{tabular}{|c|c|c|c|c|c|c|c|c|c|}
\hline \multirow[t]{2}{*}{ Predictors } & \multicolumn{3}{|c|}{ Model 1} & \multicolumn{3}{|c|}{ Model 2} & \multicolumn{3}{|c|}{ Model 3} \\
\hline & OR & $95 \% \mathrm{CI}$ & $p$-value & OR & $95 \% \mathrm{CI}$ & $p$-value & OR & $95 \% \mathrm{CI}$ & $p$-value \\
\hline Underweight & .205 & $.136-.309$ & .000 & .208 & $.138-.313$ & .000 & .211 & $.140-.319$ & .000 \\
\hline Overweight & 2.017 & $1.657-2.456$ & .000 & 2.024 & $1.663-2.465$ & .000 & 2.028 & $1.664-2.470$ & .000 \\
\hline Obesity & 2.309 & $1.694-3.148$ & .000 & 2.291 & $1.680-3.124$ & .000 & 2.345 & $1.718-3.202$ & .000 \\
\hline Depressive symptoms & - & - & - & 1.087 & $1.002-1.180$ & .046 & 1.037 & $.952-1.129$ & .405 \\
\hline Peer victimization & - & - & - & - & - & - & 1.193 & $1.091-1.305$ & .000 \\
\hline Body discrimination & 1.700 & $1.405-2.056$ & .000 & 1.595 & $1.306-1.949$ & .000 & 1.367 & $1.102-1.695$ & .004 \\
\hline Cox \& Snell $\mathrm{R}^{2}$ & \multicolumn{3}{|c|}{$.085 / .094^{1}$} & \multicolumn{3}{|l|}{.095} & \multicolumn{3}{|l|}{.100} \\
\hline
\end{tabular}

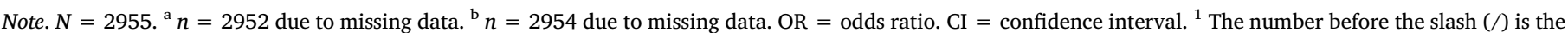
Cox \& Snell $\mathrm{R}^{2}$ for a covariates-only model; the number after the slash $(/)$ is the Cox \& Snell $\mathrm{R}^{2}$ for Model 1 .

Table 3

Linear Regression Predicting Restrained eating from Body Discrimination (Model 1) and Controlling for Depressive Symptoms (Model 2) and Peer Victimization (Model 3).

\begin{tabular}{lllllll}
\hline Predictor & Model 1 & \multicolumn{3}{c}{ Model 2 } & \multicolumn{2}{l}{ Model 3 } \\
\cline { 2 - 7 } & $\beta$ & $p$-value & $\beta$ & $p$-value & B & $p$-value \\
\hline Gender & .211 & .000 & .178 & .000 & .186 & .000 \\
Age & .002 & .907 & -.003 & .880 & -.002 & .920 \\
Indigenous status & -.022 & .200 & -.015 & .379 & -.015 & .356 \\
Household income & -.019 & .281 & -.004 & .790 & -.003 & .880 \\
Body mass index & & & & & & \\
$\quad$ Underweight & -.086 & .000 & -.077 & .000 & -.076 & .000 \\
$\quad$ Overweight & .114 & .000 & .116 & .000 & .116 & .000 \\
$\quad$ Obesity & .117 & .000 & .110 & .000 & .112 & .000 \\
Depressive symptoms & - & - & .228 & .000 & .211 & .000 \\
Peer victimization & - & - & - & - & .061 & .002 \\
Body discrimination & .213 & .000 & .141 & .000 & .119 & .000 \\
Adjusted R ${ }^{2}$ & $.091 / .135^{1}$ & & .180 & & .182 & \\
\hline
\end{tabular}

Note. $N=2955 . \beta=$ standardized beta coefficient from linear regression. ${ }^{1}$ The number before the slash $(/)$ is the Adjusted $\mathrm{R}^{2}$ for a covariates-only model; the number after the slash $(/)$ is the Adjusted $\mathrm{R}^{2}$ for Model 1 .

Although adolescents who were female or with overweight or obesity tended to report more dieting behaviors, none of the associations was moderated by gender or BMI category: The association between body discrimination and each of the health-risk behaviors was similar across males and females and across BMI categories. The one exception was for restrained eating: gender moderated its relation with body discrimination $(\beta=0.32, p<01)$. Follow-up analyses indicated that although significant for both genders, the relation was stronger among females $(\beta=.17, p<.01)$ than males $(\beta=0.08, p<.01)$.

\section{Discussion}

In a large sample of adolescents, discrimination based on the body was associated with eating-related behaviors and substance use. The association with eating behavior was independent of peer victimization whereas the association with substance use was generally accounted for by the overlap with more general forms of peer harassment. This pattern suggests that the health-risk behaviors closest to the content of the victimization had the strongest and most independent associations.

Disordered eating-related behavior is one of the most consistent correlates of victimization related to appearance and the body in both adolescence and adulthood. Individuals who experience weight discrimination, for example, tend to binge eat, skip meals, and use unhealthy methods of weight control (Vartanian \& Porter, 2016). Bodybased teasing has likewise been associated with increased dieting behavior (Chisuwa-Hayami \& Haruki, 2017), and individuals with clinical eating disorders are more likely to report a history of being teased for their appearance than healthy controls (Lie et al., 2019). Experimental evidence further suggests that exposure to weight stereotypes and stigma-related experiences increase caloric consumption. For example, women who perceived themselves as overweight ate more after exposure to a stigmatizing experience than after a neutral experience (Major, Hunger, Bunyan, \& Miller, 2014). Evidence from related literatures supports this association in adolescence. Adolescents who were teased because of their weight, for example, report more binge eating (Neumark-Sztainer et al., 2002). Other forms of discrimination have also been associated with overeating, which suggests that this type of behavior is not limited weight discrimination (Sutin, Robinson, Daly, \& Terracciano, 2016) but rather may be a common mechanism for coping with such experiences.

Unhealthy dieting behavior is likely to be one mechanism that contributes to the weight gain associated with discrimination. In middle and older adulthood, individuals who experience unfair treatment on the basis of their body weight gain more weight over time (Jackson, Beeken, \& Wardle, 2014) and are at greater risk of obesity (Sutin \& Terracciano, 2013). In young adulthood, weight stigma is associated with weight gain, likely in part due to rigid restraint eating behaviors (Wellman, Araiza, Newell, \& McCoy, 2018). In childhood, girls who are labeled "fat" by family members face a greater risk of obesity across adolescence (Hunger \& Tomiyama, 2014). Unhealthy dieting behaviors have likewise been implicated in weight gain (Neumark-Sztainer, Wall, Story, \& Standish, 2012). Although such behaviors may be effective at reducing weight in the short term, they are often hard (and dangerous) to maintain (Pearson et al., 2017) and result in the opposite of the desired effect over time. The experience of body discrimination may lead adolescents to engage in risky dieting-related behaviors that ultimately contribute to greater weight gain over time. In contrast to dieting behaviors, physical activity tends to be a healthier weightmanagement behavior. Although previous research suggests that weight discrimination is related to motivation to avoid physical activity (Vartanian \& Shaprow, 2008) and lower self-efficacy for physical activity (Pearl et al., 2015), the present research suggests that body discrimination is associated with greater engagement in exercise behavior to manage weight.

In contrast to the dieting behaviors, the associations between body discrimination and smoking and marijuana use in the current study were not independent of more general experiences with peer victimization. Peer victimization has been associated consistently with greater engagement in substances from cigarettes to inhalants in adolescence (Tharp-Taylor, Haviland, \& D'Amico, 2009; Turner et al., 2018). The association between peer victimization and substance use does not end in adolescence but extends to adulthood as well (Takizawa et al., 2014). Weight discrimination reported in adulthood has likewise been associated with use of illegal substances (Sutin \& Terracciano, 2017). The present research indicates, however, that at least in adolescence, that the association between body discrimination and substance use overlaps with more general experiences with peer victimization.

There was little evidence that any of the associations were moderated by either gender or BMI category. Research on the harmful effects of weight stigma has focused traditionally on women (Major et al., 
Table 4

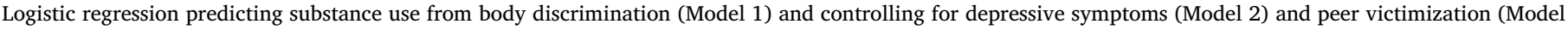
3).

\begin{tabular}{|c|c|c|c|c|c|c|c|c|c|}
\hline \multirow[t]{2}{*}{ Predictors } & \multicolumn{3}{|l|}{ Model 1} & \multicolumn{3}{|c|}{ Model 2} & \multicolumn{3}{|c|}{ Model 3} \\
\hline & OR & $95 \% \mathrm{CI}$ & $p$-value & OR & $95 \% \mathrm{CI}$ & $p$-value & OR & $95 \% \mathrm{CI}$ & $p$-value \\
\hline \multicolumn{10}{|l|}{ Cigarette Smoking } \\
\hline Gender & 1.233 & $.960-1.584$ & .101 & 1.063 & $.822-1.375$ & .642 & 1.211 & $.929-1.578$ & .156 \\
\hline Age & 1.395 & $1.086-1.792$ & .009 & 1.350 & $1.048-1.740$ & .020 & 1.375 & $1.064-1.778$ & .015 \\
\hline Indigenous status & 2.132 & $1.092-4.165$ & .027 & 2.465 & $1.247-4.875$ & .009 & 2.392 & $1.193-4.798$ & .014 \\
\hline Household income & .796 & $.691-.916$ & .002 & .828 & $.715-.958$ & .011 & .842 & $.723-.980$ & .026 \\
\hline \multicolumn{10}{|l|}{ Body mass index } \\
\hline Underweight & .449 & $.265-.940$ & .031 & .541 & $.286-1.035$ & .059 & .587 & $.309-1.113$ & .103 \\
\hline Overweight & .794 & $.572-1.101$ & .167 & .802 & $.575-1.118$ & .193 & .805 & $.575-1.126$ & .205 \\
\hline Obesity & .994 & $.629-1.571$ & .980 & .906 & $.566-1.448$ & 679 & .935 & $.580-1.508$ & .783 \\
\hline Depressive symptoms & - & - & - & 1.550 & $1.388-1.730$ & .000 & 1.408 & $1.251-1.586$ & .000 \\
\hline Peer victimization & - & - & - & - & - & - & 1.520 & $1.341-1.722$ & .000 \\
\hline Body discrimination & 2.851 & $2.191-3.709$ & .000 & 1.995 & $1.505-2.644$ & .000 & 1.334 & $.976-1.822$ & .070 \\
\hline Cox \& Snell $\mathrm{R}^{2}$ & $.011 / .030^{1}$ & & .049 & & & .062 & & & \\
\hline \multicolumn{10}{|l|}{ Alcohol } \\
\hline Gender & 1.004 & $.866-1.165$ & .955 & .946 & $.813-1.099$ & .467 & 1.019 & $.874-1.188$ & .809 \\
\hline Age & 1.456 & $1.251-1.694$ & .000 & 1.452 & $1.246-1.691$ & .000 & 1.472 & $1.262-1.716$ & .000 \\
\hline Indigenous status & 1.378 & $.807-2.352$ & .240 & 1.446 & $.845-2.473$ & .178 & 1.411 & $.823-2.421$ & .211 \\
\hline Household income & .968 & $.877-1.068$ & .513 & .984 & $.891-1.086$ & .744 & .995 & $.900-1.099$ & .914 \\
\hline \multicolumn{10}{|l|}{ Body mass index } \\
\hline Underweight & .642 & $.472-.873$ & .005 & .659 & $.484-.896$ & .008 & .677 & $.497-.924$ & .014 \\
\hline Overweight & 1.083 & $.893-1.314$ & .416 & 1.088 & $.897-1.321$ & .391 & 1.088 & $.895-1.323$ & .397 \\
\hline Obesity & .894 & $.662-1.206$ & .462 & .868 & $.642-1.174$ & .359 & .891 & $.657-1.209$ & .458 \\
\hline Depressive symptoms & - & - & - & 1.242 & $1.143-1.350$ & .000 & 1.147 & $1.053-1.250$ & .002 \\
\hline Peer victimization & - & - & - & - & - & - & 1.367 & $1.247-1.499$ & .000 \\
\hline Body discrimination & 2.058 & $1.701-2.490$ & .000 & 1.763 & $1.444-2.153$ & .000 & 1.365 & $1.102-1.692$ & .004 \\
\hline Cox \& Snell $\mathrm{R}^{2}$ & $.011 / .031^{1}$ & & .040 & & & .055 & & & \\
\hline \multicolumn{10}{|l|}{ Marijuana } \\
\hline$\overline{\text { Gender }}$ & .882 & $.646-1.185$ & .404 & .752 & $.555-1.019$ & .066 & .846 & $.620-1.155$ & .293 \\
\hline Age & 1.908 & $1.422-2.560$ & .000 & 1.837 & $1.365-2.472$ & .000 & 1.885 & $1.396-2.544$ & .000 \\
\hline Indigenous status & 3.324 & $1.703-6.486$ & .000 & 3.901 & $1.977-7.699$ & .000 & 3.927 & $1.974-7.814$ & .000 \\
\hline Household income & .729 & $.628-.847$ & .000 & .756 & $.648-.883$ & .001 & .764 & $.651-.897$ & .001 \\
\hline \multicolumn{10}{|l|}{ Body mass index } \\
\hline Underweight & .710 & $.374-1.348$ & .295 & .777 & $.406-1.484$ & .444 & .841 & $.440-1.609$ & .601 \\
\hline Overweight & .621 & $.412-.935$ & .023 & .632 & $.418-.955$ & .030 & .626 & $.414-.954$ & .029 \\
\hline Obesity & .584 & $.314-1.086$ & .089 & .533 & $.283-1.002$ & .051 & .537 & $.283-1.018$ & .057 \\
\hline Depressive symptoms & - & - & - & 1.577 & $1.390-1.790$ & .000 & 1.434 & $1.250-1.645$ & .000 \\
\hline Peer victimization & - & - & - & - & - & - & 1.500 & $1.301-1.728$ & .000 \\
\hline Body discrimination & 2.306 & $1.680-3.165$ & .000 & 1.591 & $1.135-2.232$ & .007 & 1.064 & $.733-1.545$ & .742 \\
\hline Cox \& Snell $\mathrm{R}^{2}$ & $.015 / .024^{1}$ & & .038 & & & .048 & & & \\
\hline
\end{tabular}

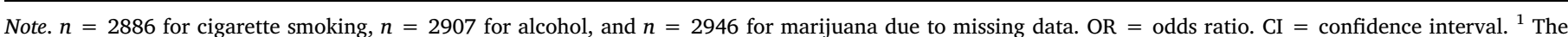
number before the slash $(/)$ is the Cox $\&$ Snell $\mathrm{R}^{2}$ for a covariates-only model; the number after the slash $(/)$ is the Cox \& Snell $\mathrm{R}^{2}$ for Model 1 .

2014; Mensinger, Calogero, \& Tylka, 2016), although many studies have recently found discrimination to be equally harmful across demographic groups, including across gender (Sutin et al., 2016; Sutin \& Terracciano, 2017). Thus, even if there are differences in the prevalence of weight discrimination (Dutton et al., 2014) and disordered eatingrelated behaviors (Striegel-Moore et al., 2009), the relation between the two are similar across gender. The present research was primarily consistent with the view that the correlates of body discrimination do not differ by gender. There was, however, one exception: the association between body discrimination and restrained eating was stronger among females than males. Women may restrain their eating more than men as a means of weight control as a result of experiencing unfair treatment because of their weight. There was no evidence that body discrimination was more or less harmful for the outcomes by BMI category.

The present research has several strengths, including a large sample of adolescents, measured adiposity, and assessment of several healthrisk behaviors. There are also some limitations that could be addressed in future research. For example, all of the outcomes were self-reported. The adolescents reported on each of the outcomes straight into a computer to protect their privacy, but it is still possible that participants did not want to report engagement in unhealthy and/or illegal behaviors or did so inaccurately. In addition, the prevalence of the most harmful dieting behaviors was quite low. It is encouraging that relatively few of the adolescent participants engaged in such behavior. Although the low prevalence may limit the statistical power to detect robust effects, the results are consistent with the broader literature on discrimination and extreme dieting behaviors (Vartanian \& Porter, 2016). In addition, it may be the case that body discrimination is one expression of peer victimization (i.e., a mediator) that cannot be separated from it. Participants could have included body-based discrimination in their reports of general victimization. Future research could use more detailed measures to better disentangle body-based discrimination from other forms of peer victimization. Still, the correlation between body discrimination and peer victimization was only moderate $(r=0.44)$ likely due, in part, to the broader nature of the body discrimination measure that could include discrimination by family members and other adults not captured on the peer victimization measure. Further, it maintained strong, independent associations with the dieting-related behaviors controlling for peer victimization. This pattern indicates that it is detecting risk over and above reports of general victimization. Finally, we controlled for depressive symptoms because of the potential overlap with both the measures of victimization and the outcomes. It may also be the case that depressive symptoms are a mediator of these associations. Future research could address this issue with longitudinal data to disentangle the temporal ordering 
and identify mechanisms that contribute to these associations. Despite these limitations, the present research indicates that body discrimination is associated with harmful health behaviors at least as early as age 14 , and the associations are independent of more general forms of peer victimization.

\section{Ethical statement}

The Australian Institute of Family Studies Ethics Committee approved data collection for LSAC and written informed consent for each studied family was obtained before family members were asked any questions. The Institutional Review Board at the Florida State University also approved analysis of these data.

\section{Acknowledgement}

The Longitudinal Study of Australian Children (LSAC) is funded by the Commonwealth Department of Families, Community Services and Indigenous Affairs. No direct funds from the LSAC study supported this research.

\section{Appendix A. Supplementary data}

Supplementary data to this article can be found online at https:// doi.org/10.1016/j.appet.2020.104689.

\section{Author contributions}

ARS conceived the idea for the study and secured and analyzed the data. YS, ER, MD, and AT provided critical feedback on the conceptualization, analysis, and interpretation of the findings. ARS drafted the manuscript. All authors provided feedback and edited the manuscript and all authors approved the final manuscript.

\section{References}

Angold, A., Costello, E. J., Messer, S. C., Pickles, A., Winder, F., \& Silver, D. (1995). Development of a short questionnaire for use in epidemiological studies of depression in children and adolescents. International Journal of Methods in Psychiatric Research, 5, 237-249.

Brockenbrough, K. K., Cornell, D. G., \& Loper, A. B. (2002). Aggressive attitudes among victims of violence at school. Education \& Treatment of Children, 25, 273-287.

Chisuwa-Hayami, N., \& Haruki, T. (2017). Associations of body-related teasing with weight status, body image, and dieting behavior among Japanese adolescents. Health Promotion Perspectives, 7, 80-87. https://doi.org/10.15171/hpp.2017.15.

Copeland, W. E., Bulik, C. M., Zucker, N., Wolke, D., Lereya, S. T., \& Costello, E. J. (2015). Does childhood bullying predict eating disorder symptoms? A prospective, longitudinal analysis. International Journal of Eating Disorders, 48, 1141-1149. https://doi. org/10.1002/eat.22459.

Duncan, N., Zimmer-Gembeck, M. J., \& Furman, W. (2019). Sexual harassment and appearance-based peer victimization: Unique associations with emotional adjustment by gender and age. Journal of Adolescence, 75, 12-21. https://doi.org/10.1016/j. adolescence.2019.06.016.

Dutton, G. R., Lewis, T. T., Durant, N., Halanych, J., Kiefe, C. I., Sidney, S., et al. (2014). Perceived weight discrimination in the CARDIA study: Differences by race, sex, and weight status. Obesity (Silver Spring), 22, 530-536. https://doi.org/10.1002/oby. 20438.

Earnshaw, V. A., Elliott, M. N., Reisner, S. L., Mrug, S., Windle, M., Emery, S. T., et al. (2017). Peer victimization, depressive symptoms, and substance use: A longitudinal analysis. Pediatrics, 139, e20163426. https://doi.org/10.1542/peds.2016-3426.

Greenleaf, C., Petrie, T. A., \& Martin, S. B. (2014). Relationship of weight-based teasing and adolescents' psychological well-being and physical health. Journal of School Health, 84, 49-55. https://doi.org/10.1111/josh.12118.

Hunger, J. M., \& Tomiyama, A. J. (2014). Weight labeling and obesity: A longitudinal study of girls aged 10 to 19 years. JAMA Pediatrics, 168, 579-580. https://doi.org/10. 1001/jamapediatrics.2014.122.

Jackson, S. E., Beeken, R. J., \& Wardle, J. (2014). Perceived weight discrimination and changes in weight, waist circumference, and weight status. Obesity (Silver Spring), 22, 2485-2488. https://doi.org/10.1002/oby.20891.

Klinck, M., Vannucci, A., Fagle, T., \& Ohannessian, C. M. (2020). Appearance-related teasing and substance use during early adolescence. Psychology of Addictive Behaviors. https://doi.org/10.1037/adb0000563.

Kuczmarski, R. J., Ogden, C. L., Grummer-Strawn, L. M., Flegal, K. M., Guo, S. S., Wei, R., et al. (2000). CDC growth charts: United States. Advance Data, 314, 1-27.

Lee, H., Son, I., Yoon, J., \& Kim, S. S. (2017). Lookism hurts: Appearance discrimination and self-rated health in South Korea. International Journal for Equity in Health, 16, 204. https://doi.org/10.1186/s12939-017-0678-8.

Lie, S., Rø, Ø., \& Bang, L. (2019). Is bullying and teasing associated with eating disorders? A systematic review and meta-analysis. International Journal of Eating Disorders, 52, 497-514. https://doi.org/10.1002/eat.23035.

Major, B., Hunger, J. M., Bunyan, D. P., \& Miller, C. T. (2014). The ironic effects of weight stigma. Journal of Experimental Social Psychology, 51, 74-80. https://doi.org/10. 1016/j.jesp.2013.11.009.

McAra, L., \& McVie, S. (2010). Youth crime and justice: Key messages from the Edinburgh study of youth transitions and crime. Criminology and Criminal Justin, 10, 179-209. https://doi.org/10.1177/1748895809360971.

Mensinger, J. L., Calogero, R. M., \& Tylka, T. L. (2016). Internalized weight stigma moderates eating behavior outcomes in women with high BMI participating in a healthy living program. Appetite, 102, 32-43. https://doi.org/10.1016/j.appet.2016. 01.033 .

Menzel, J. E., Schaefer, L. M., Burke, N. L., Mayhew, L. L., Brannick, M. T., \& Thompson, J. K. (2010). Appearance-related teasing, body dissatisfaction, and disordered eating: A meta-analysis. Body Image, 7, 261-270. https://doi.org/10.1016/j.bodyim.2010. 05.004.

Moore, S. E., Norman, R. E., Suetani, S., Thomas, H. J., Sly, P. D., \& Scott, J. G. (2017) Consequences of bullying victimization in childhood and adolescence: A systematic review and meta-analysis. World Journal of Psychiatry, 7, 60-76. https://doi.org/10. 5498/wjp.v7.i1.60.

Neumark-Sztainer, D., Falkner, N., Story, M., Perry, C., Hannan, P. J., \& Mulert, S. (2002) Weight-teasing among adolescents: Correlations with weight status and disordered eating behaviors. International Journal of Obesity, 26, 123-131. https://doi.org/10. 1038/sj/ijo/0801853.

Neumark-Sztainer, D., Wall, M., Story, M., \& Standish, A. R. (2012). Dieting and unhealthy weight control behaviors during adolescence: Associations with 10-year changes in body mass index. Journal of Adolescent Health, 50, 80-86. https://doi.org/ 10.1016/j.jadohealth.2011.05.010.

Pearl, R. L., Puhl, R. M., \& Dovidio, J. F. (2015). Differential effects of weight bias experiences and internalization on exercise among women with overweight and obesity. Journal of Health Psychology, 20, 1626-1632. https://doi.org/10.1177/ 1359105313520338

Pearson, C. M., Miller, J., Ackard, D. M., Loth, K. A., Wall, M. M., Haynos, A. F., et al (2017). Stability and change in patterns of eating disorder symptoms from adolescence to young adulthood. International Journal of Eating Disorders, 50, 748-757. https://doi.org/10.1002/eat.22692.

Pont, S. J., Puhl, R., Cook, S. R., \& Slusser, W. Section on Obesity, and The Obesity Society. (2017). Stigma experienced by children and adolescents with obesity. Pediatrics, 140, e20173034. https://doi.org/10.1542/peds.2017-3034.

Priesman, E., Newman, R., \& Ford, J. A. (2017). Bullying victimization, binge drinking, and marijuana use among adolescents: Results from the 2013 National Youth Risk Behavior Survey. Journal of Psychoactive Drugs, 1-10. https://doi.org/10.1080/ 02791072.2017.1371362.

Puhl, R. M., Andreyeva, T., \& Brownell, K. D. (2008). Perceptions of weight discrimination: Prevalence and comparison to race and gender discrimination in America. International Journal of Obesity, 32. https://doi.org/10.1038/ijo.2008.22.

Puhl, R. M., \& King, K. M. (2013). Weight discrimination and bullying. Best Practice \& Research Clinical Endocrinology \& Metabolism, 27, 117-127. https://doi.org/10.1016/ j.beem.2012.12.002.

Selzer, R., Hamill, C., Bowes, G., \& Patton, G. (1996). The branched eating disorders test: Validity in a nonclinical population. International Journal of Eating Disorders, 20, 57-64. https://doi.org/10.1002/(SICI)1098-108X(199607)20:1 < 57::AID-EAT7 > 3. $0 . \mathrm{CO} ; 2-3$.

Spahlholz, J., Pabst, A., Riedel-Heller, S. G., \& Luck-Sikorski, C. (2016). Coping with perceived weight discrimination: Testing a theoretical model for examining the relationship between perceived weight discrimination and depressive symptoms in a representative sample of individuals with obesity. International Journal of Obesity, 40, 1915-1921. https://doi.org/10.1038/ijo.2016.164.

Striegel-Moore, R. H., Rosselli, F., Perrin, N., DeBar, L., Wilson, G. T., May, A., et al. (2009). Gender difference in the prevalence of eating disorder symptoms. International Journal of Eating Disorders, 42, 471-474. https://doi.org/10.1002/eat. 20625.

Sutin, A., Robinson, E., Daly, M., \& Terracciano, A. (2016). Weight discrimination and unhealthy eating-related behaviors. Appetite, 102, 83-89. https://doi.org/10.1016/j. appet.2016.02.016.

Sutin, A. R., Stephan, Y., Carretta, H., \& Terracciano, A. (2015). Perceived discrimination and physical, cognitive, and emotional health in older adulthood. American Journal of Geriatric Psychiatry, 23, 171-179. https://doi.org/10.1016/j.jagp.2014.03.007.

Sutin, A. R., Stephan, Y., \& Terracciano, A. (2015). Weight discrimination and risk of mortality. Psychological Science, 26, 1803-1811. https://doi.org/10.1177/ 0956797615601103.

Sutin, A. R., \& Terracciano, A. (2013). Perceived weight discrimination and obesity. PloS One, 8, e70048. https://doi.org/10.1371/journal.pone.0070048.

Sutin, A. R., \& Terracciano, A. (2017). Perceived weight discrimination and high-risk health-related behaviors. Obesity, 25, 1183-1186. https://doi.org/10.1002/oby. 21845.

Takizawa, R., Danese, A., Maughan, B., \& Arseneault, L. (2015). Bullying victimization in childhood predicts inflammation and obesity at mid-life: A five-decade birth cohort study. Psychological Medicine, 45, 2705-2715. https://doi.org/10.1017/ S0033291715000653.

Takizawa, R., Maughan, B., \& Arseneault, L. (2014). Adult health outcomes of childhood bullying victimization: Evidence from a five-decade longitudinal British birth cohort. American Journal of Psychiatry, 171, 777-784. https://doi.org/10.1176/appi.ajp. 
2014.13101401.

Tharp-Taylor, S., Haviland, A., \& D'Amico, E. J. (2009). Victimization from mental and physical bullying and substance use in early adolescence. Addictive Behaviors, 34, 561-567. https://doi.org/10.1016/j.addbeh.2009.03.012.

Tomiyama, A. J. (2014). Weight stigma is stressful. A review of evidence for the Cyclic Obesity/Weight-Based Stigma model. Appetite, 82, 8-15. https://doi.org/10.1016/j. appet.2014.06.108.

Turner, S., Taillieu, T., Fortier, J., Salmon, S., Cheung, K., \& Afifi, T. O. (2018). Bullying victimization and illicit drug use among students in grades 7 to 12 in Manitoba, Canada: A cross-sectional analysis. Canadian Journal of Public Health, 109, 183-194. https://doi.org/10.17269/s41997-018-0030-0.

Vartanian, L. R., \& Porter, A. M. (2016). Weight stigma and eating behavior: A review of the literature. Appetite, 102, 3-14. https://doi.org/10.1016/j.appet.2016.01.034.

Vartanian, L. R., \& Shaprow, J. G. (2008). Effects of weight stigma on exercise motivation and behavior: A preliminary investigation among college-aged females. Journal of Health Psychology, 13, 131-138.

Vivolo-Kantor, A. M., Martell, B. N., Holland, K. M., \& Westby, R. (2014). A systematic review and content analysis of bullying and cyber-bullying measurement strategies. Aggression and Violent Behavior, 19, 423-434. https://doi.org/10.1016/j.avb.2014.06. 008.

Wellman, J. D., Araiza, A. M., Newell, E. E., \& McCoy, S. K. (2018). Weight stigma facilitates unhealthy eating and weight gain via fear of fat. Stigma Health, 3, 186-194. https://doi.org/10.1037/sah0000088.

Zimmer-Gembeck, M. J., Webb, H. J., Farrell, L. J., \& Waters, A. M. (2018). Girls' and boys' trajectories of appearance anxiety from age 10 to 15 years are associated with earlier maturation and appearance-related teasing. Developmental Psychopathology, 30, 337-350. https://doi.org/10.1017/S0954579417000657. 